\title{
¿Iguales en qué y cómo? \\ Una revisión de las propuestas realizadas desde la teoría de la justicia al campo de la educación*
}

\author{
Alejandro Montes \\ Lluís Parcerisa \\ Universitat Autònoma de Barcelona. Globalització, Educació i Polítiques Socials (GEPS) \\ alejandro.montes@uab.cat; lluis.parcerisa@uab.cat
}

Recibido: 16-01-2015

Aceptado: 15-04-2016

\section{Resumen}

El presente artículo hace una revisión de las principales teorías de la justicia aplicadas al campo educativo. En primer lugar, se muestran las aportaciones más importantes de la teoría social normativa al campo de la política educativa, enfatizando aquellas posturas que han sido dominantes históricamente: la meritocracia y la teoría de la justicia de Rawls. En segundo lugar, se exponen las limitaciones y las principales críticas realizadas a las mismas. Finalmente, se presentan las aportaciones de las nuevas perspectivas centradas en dimensiones hasta el momento obviadas, lo que llamamos «justicia postdistributiva», que complementan a las teorías anteriores. En definitiva, con este trabajo, se pretende sistematizar las diversas miradas existentes en el campo de la teoría de la justicia social en educación, sus implicaciones en términos de igualdad y equidad, así como los criterios normativos que se desprenden de estas, de necesaria consideración a la hora de diseñar y evaluar políticas educativas que pretendan hacer frente a las desigualdades.

Palabras clave: justicia distributiva; igualdad de oportunidades; equidad educativa; teorías de la justicia; políticas educativas; teorías normativas; políticas de representación; políticas de reconocimiento

* Queremos agradecer a Aina Tarabini sus aportaciones en forma de comentarios y críticas. 
Abstract. Equal in What and How?: A Review of the Proposals From the Theory of Justice to the Field of Education

This article reviews the application of the main theories of justice to the field of education. First, the main contributions of social normative theory to the field of educational policy are examined, emphasizing those positions that have historically dominated this field: meritocracy and Rawl's theory of justice. Secondly, the limitations and main criticisms of both are examined. Finally, the contributions of new perspectives focusing on dimensions overlooked until now - what we call "postdistributive justice" - that complement the above theories are presented. This paper aims to systematize the different approaches that have existed and exist in the field of theory of social justice in education, their implications in terms of equality and equity, and the normative criteria arising from them, which must be considered when designing and evaluating educational policies that seek to address inequalities.

Keywords: distributive justice; equal opportunities; educational equity; theories of justice; education policy; normative theories; representation policies; recognition policies

\section{Sumario}

Introducción

2. Las corrientes clásicas de la justicia social y su aplicación en el campo de la educación

3. Limitaciones y críticas de la justicia como compensación
4. Justicia postdistributiva y equidad educativa

5. Conclusiones

Referencias bibliográficas

\section{Introducción}

Tal y como muestra la literatura existente, la relación entre educación y desigualdad tiene un carácter bidireccional (Bonal y Tarabini, 2010). En este sentido, toda incidencia en el campo de las políticas educativas, de una forma u otra, repercutirá en el crecimiento, el mantenimiento o la disminución de las desigualdades sociales (Tarabini, 2006).

Sin embargo, no podemos asumir que, en el transcurso de la modernidad, y de la evolución que esta ha comportado en las instituciones sociales, la concepción sobre la funcionalidad de la educación se haya mantenido estanca. Postulados como la igualdad de oportunidades de acceso se han visto inevitablemente superados, aportando a su vez nuevos criterios que explican y corrigen el surgimiento de desigualdades, añadiendo más dimensiones al debate sobre la justicia y la igualdad.

Esto sugiere una serie de interrogantes: ¿Cómo se puede determinar si un sistema educativo es justo? ¿Implica ello que este sea igualitario? ¿En qué dimensiones es necesario hacer un énfasis especial para que esta justicia se dé efectivamente? ¿Qué papel desempeña la política educativa en esa cuestión? En el transcurso del presente artículo, intentaremos responder a dichas cuestiones con el objetivo de discernir qué mecanismos entroncan la justicia educativa 
y cuáles son los grandes retos para la justicia social y la equidad educativa de aquí en adelante. Para ello, analizaremos las diferentes propuestas generadas a nivel teórico sobre la justicia social aplicada al campo educativo. Finalmente, realizaremos una reflexión en torno a las necesidades de reconocimiento y representación en el campo de la educación.

\section{Las corrientes clásicas de la justicia social y su aplicación en el campo de la educación}

\subsection{Igualdad y educación. De la meritocracia a la justicia como equidad}

El debate sobre el principio meritocrático en educación tiene un largo recorrido. En la historia reciente, el autor que introdujo el debate alrededor de esta cuestión fue Michael Young (1961), con su conocida obra The rise of the meritocracy. La etimología del concepto de meritocracia nos remite a dos componentes: por un lado, está formada por el vocablo latín meritum, es decir, por la acción o las acciones que ha desarrollado una persona y que la hacen merecedora de recibir un premio o un castigo. Por otro lado, encontramos la idea de kratos, que se relaciona con la idea de poder y que, en griego, generalmente se utiliza para mencionar al Estado (García Cívico, 2006). En palabras de Puyol (2007: 170):

[...] la meritocracia es un sistema social basado en la aristocracia del talento y no en alguna forma de justicia democrática o igualitaria; consiste en distribuir los trabajos, los cargos y las recompensas sociales y económicas de acuerdo a las cualidades y calificaciones individuales.

Según Young (1961: 110), en la época en que escribió su obra, se creía que se vivía una especie de «edad de oro de la igualdad». La principal razón para pensar así era que, durante aquella época, se vivía una disputa entre dos principios antagónicos que se utilizan para legitimar el acceso a posiciones de poder, a saber: el parentesco (o la herencia) y el mérito. Ambos principios disfrutaban de una amplia aceptación social. No obstante, la aplicación simultánea de estos principios, lejos de conducir a una mayor igualdad, contribuyó a exacerbar la exclusión social, restringiendo aún más el acceso a las posiciones de mayor poder a aquellos nacidos en entornos privilegiados, que pudieran demostrar que disponían de un mayor número de méritos individuales. En palabras de Bolívar (2005), el modelo meritocrático se sustenta en la idea de que la distribución de los bienes sociales o educativos se debe realizar de acuerdo con los méritos alcanzados a nivel individual, de manera que el mérito de cada individuo se convierte en un principio básico orientador de la idea de justicia. Sin embargo, Young (1961) considera que, si no se toma en consideración el contexto de los individuos, es decir, la existencia de condiciones estructurales de desigualdad, aunque exista una igualdad formal, tanto en el trato como en los derechos y las oportunidades, estas solo servirán como mecanismo de reproducción de las desigualdades sociales. En un sentido similar, Dubet y Duru- 
Bellat cuestionan algunas de las falacias de la justicia meritocrática y señalan que la propia idea de justicia se ve afectada y/o alterada por las desigualdades de partida presentes en la sociedad, critican el supuesto según el cual la escuela es un espacio donde existe una pura o verdadera igualdad de oportunidades y destacan la dificultad de articular una definición objetiva del mérito (Dubet y Duru-Bellat, 2004; citados por Bolívar, 2005).

Es por ello que, en el marco de la sociedad del conocimiento, la educación se ha convertido en uno de los bienes principales para el desarrollo de las aptitudes, de las habilidades y de los conocimientos necesarios para ejercer una vida social plena. A su vez, la educación no puede considerarse como un derecho individual, sino que se trata de un derecho social con beneficios que repercuten en el bienestar de la comunidad, el cual debe ser orientado por parte de las políticas públicas pertinentes (Tarabini, 2006). En este sentido, según Walker y Unterhalter (2007), podemos distinguir tres tipos de valores de la educación (intrínsecos, instrumentales y posicionales). En primer lugar, encontramos el valor intrínseco, que se refiere a la contribución de la educación en relación con el desarrollo personal de cada individuo. En segundo lugar, el valor instrumental está vinculado al desarrollo que genera la educación (valor intrínseco) y los beneficios que este genera a la hora de acceder a determinados bienes colectivos. Por último, encontramos el valor posicional, que se refiere a las ventajas relativas de las que disfruta el individuo gracias al valor adquirido mediante la consecución de acreditaciones educativas (Gallego Duque, 2014). $\mathrm{Al}$ mismo tiempo, como institución social, puede acontecer como instrumento básico de legitimación y reproducción del statu quo. Es en este contexto donde surge la importancia de aplicar una dimensión de justicia en la educación, precisamente con el objetivo de explicitar el posicionamiento deseable de la institución educativa en el debate en torno a su papel como reproductora o transformadora social.

Según Bolívar (2012), autores como Bobbio (1993) han señalado de manera acertada que este debate resulta muy interesante cuando se abordan las cuestiones vinculadas al ámbito en el que debe haber igualdad (¿en qué?) y entre los sujetos entre los que esta se hace efectiva (¿entre quiénes?). A menudo, los conceptos subyacentes a la teoría social normativa se acostumbran a emplear de manera totalmente acrítica, utilizando indistintamente los conceptos de justicia, equidad e igualdad. La definición de igualdad más sencilla sería la que subyace de la idea "de entre todos en todo", es decir, como aquel tipo de igualdad formal de los ciudadanos de la que se habla en las constituciones de los estados o en los derechos, aunque lo cierto es que difícilmente se puede sostener que la igualdad se dé en todos los ámbitos. De manera estricta, podemos entender que «la igualdad en el tratamiento se basa en la regla simple de distribuir fracciones iguales de recursos a todas las personas» (Bolívar, 2012: 13).

En el campo de la docencia, este concepto a menudo se ha traducido por igualdad de oportunidades. Por ello, la preocupación básica de la política pedagógica al respecto ha sido la de facilitar el acceso educativo a toda la pobla- 
ción, sin distinción de clase, género o etnia. Bajo este punto de vista, como pone de relieve Fernández Enguita (1998), se igualó el concepto de igualdad al de igualdad de oportunidad de acceso y se focalizó en la lucha contra las desigualdades de partida de carácter socioeconómico y cultural, tanto propias como familiares. El resultado fue la expansión de los sistemas educativos en la sociedad moderna actual. Desde esta lógica, llegados a dicha igualdad «simple», los méritos serían los encargados de producir procesos de distinción que, a la larga, generarían desigualdades justas entre los distintos individuos. La política educativa debería simplemente asegurar la provisión de esa educación, promoviendo la obligatoriedad y la universalidad, asegurando la igualdad de oportunidades de acceso.

Sin embargo, como citábamos anteriormente, el concepto de igualdad es polisémico y tiene diferentes significados, lo que provoca que tengamos que concretar si hablamos de igualdad en el acceso a la educación, o bien en el currículo, o en los resultados (Bolívar, 2012). Siguiendo el trabajo de este autor, podemos distinguir entre diversos principios de igualdad, centrando cada uno de ellos en una dimensión de la reducción de las desigualdades. A su vez, centrarse en la obtención de alguno de estos tipos de igualdad puede suponer la aceptación, directa o indirecta, de alguna de las demás desigualdades. En primer lugar, la igualdad de oportunidades, como hemos explicado, hace referencia a dos cuestiones: «nivelar el campo de juego" y evitar las discriminaciones por condicionantes personales (Bolívar, 2012). Con el desarrollo de las teorías de la justicia, esta idea de compensación de las circunstancias personales se verá ampliada introduciendo al debate elementos de equidad. En segundo lugar, la igualdad de enseñanza hace referencia a elementos de currículo, profesorado, centro y entorno escolar. Como base previa al desarrollo de programas de discriminación positiva (es decir, aquellos programas que favorecen a los más débiles), es básico establecer una oferta y unas condiciones formativas equitativas e iguales para todos. En tercer lugar, la igualdad de conocimiento y éxito escolar hace referencia a la lucha contra la indiferencia a las diferencias. Por último, la igualdad de resultados hace referencia a la necesidad de propiciar unas mismas oportunidades sociales, actuando no solo sobre los outputs, sino también sobre los outcomes (Bolívar, 2012).

En cuanto a la noción de equidad, esta toma fuerza desde la publicación de la teoría de la justicia de Rawls (1971) y quiere ir más allá de la igualdad, suponiendo «que determinadas desigualdades, además de inevitables, deben ser tenidas en cuenta». De la anterior afirmación, se deriva que hay que superar la igualdad formal y dar un trato desigual favorable a los menos favorecidos. En educación, la concepción tradicional de la igualdad de oportunidades se centraría en cuestiones relacionadas con la igualdad relativa a la distribución de recursos, como, por ejemplo: garantizar el acceso a la escolarización. En este sentido, creemos que la concepción rawlsiana de igualdad de oportunidades supera la concepción tradicional, ya que incorpora un elemento de compensación de las desigualdades de origen (sean sociales o naturales). En política edu- 
cativa, esto se traduciría en la adopción de acciones compensatorias orientadas a la mejora de las condiciones de escolaridad y a las competencias académicas de los estudiantes menos aventajados (Bolívar, 2005).

De este modo, vemos que, en el campo de la educación, hay un debate encendido que gira alrededor de cómo se entiende la igualdad educativa, en el que se tratan, por un lado, aspectos vinculados a las insuficiencias explicativas que nos ofrece la idea de igualdad formal y, por otro, las exigencias y/o demandas de equidad por parte de los más desfavorecidos. Dicho esto, y para clarificar conceptualmente el paradigma de la igualdad y el de la equidad en el campo de la educación, podemos decir que mientras que el primero estaría vinculado a la satisfacción de una parte esencial del derecho a la educación (el derecho de acceso), en el caso del segundo, este se refiere a la consecución del éxito educativo de todo el alumnado (Bolívar, 2012: 13).

\subsection{La idea de justicia como equidad}

Uno de los objetivos al que aspiran las políticas educativas, o al menos que prometían con la llegada de la escolarización de masas, era la reducción efectiva de las desigualdades sociales.

El discurso sobre la igualdad de oportunidades (y la equidad) en el ámbito educativo ha sido muy extendido y aceptado socialmente, y cuenta con una tradición histórica bastante larga (Tedesco, 2004). En este sentido, como se mencionaba anteriormente, en el paradigma de la igualdad, los individuos deben recibir el mismo trato para que este sea considerado justo. Por otro lado, desde la perspectiva de la equidad, habrá un trato justo cuando dichas desigualdades sean reconocidas con el objetivo de atajarlas. La idea subyacente desde este paradigma es que el tratamiento desigual puede ser justo siempre y cuando beneficie a aquellos individuos más desfavorecidos.

El trabajo clásico de Rawls (1971) partiría de este segundo paradigma. En el campo educativo específicamente, la igualdad de oportunidades es un legado del primer paradigma, mientras que el segundo deja en herencia ciertas medidas de educación compensatoria, si bien, en la práctica, es difícil aplicar medidas que dejen completamente de lado parte de ambos principios, siendo la propia teoría de la justicia de Rawls un buen ejemplo de la incorporación de elementos de ambos.

En trabajos posteriores, las críticas contra la visión meritocrática y su concepción de igualdad se han hecho patentes. Para autores como Dubet (2011), la creencia en un mundo justo en el que la retribución se realiza según el esfuerzo y el mérito, se constituye en múltiples ocasiones como la justificación final de la desigualdad. En la misma línea, trabajos clásicos como los de Bernstein (1999) también pusieron de relieve que el principio de igualdad de oportunidades, por sí mismo, no funciona, ya que los alumnos desfavorecidos tenían igualmente menores oportunidades.

Con Rawls (1971), surge una concepción no utilitarista de la justicia, la cual podríamos catalogar como concepción de justicia como equidad. Para ello, 
Rawls se basa en la teoría de la elección racional ${ }^{1}$ y argumenta que, a partir del velo de la ignorancia, es decir, una vez desvinculada la preferencia o la elección de los condicionantes individuales particulares, la elección tomada será la más justa. De este modo, se acordarían de forma equitativa las nociones de justicia social, siempre bajo tres principios: el principio de igual libertad, el principio de igualdad equitativa de oportunidades y el principio de diferencia (Rawls, 1971; Tarabini, 2006).

El primer principio hace referencia a la necesidad de establecer el máximo número posible de libertades básicas de cada individuo. El segundo, al hecho de que, en una sociedad justa, se ha de tender a la igualdad, de tal manera que aquello que suceda quede bajo la responsabilidad y la elección propia y se minimicen los efectos arbitrarios. Es aquí donde establecemos vínculos con el tercer principio, ya que un sistema de igualdad de oportunidades compensatorio como el que propone Rawls no niega la desigualdad, siempre y cuando esta contribuya a beneficiar a los miembros más desaventajados de la sociedad. En esta línea, se supera el concepto de igualdad simple, ya que se interpreta que dicha situación no produciría un mayor beneficio social si no se interviniera en las desigualdades más allá del acceso. En el ámbito educativo, esto supone asumir un sistema que compense las desventajas sociales e individuales asociadas a cada individuo, aunque, ciertamente, las implicaciones de esta concepción han de ir más allá de la mera compensación, con el objetivo de formalizarse finalmente en términos de una adecuación educativa igualitaria (Satz, 2007).

A su vez, a partir de la aplicación del tercer principio, el de diferencia, se supera la idea estricta de justicia distributiva y la de justicia en la igualdad de oportunidades, mediante la ruptura con la esencia de las concepciones darwinianas de la meritocracia y su sistema de clasificación escolar. Es con la aplicación de estos principios que, sin tener en cuenta los efectos sociales ni políticos de la distribución, es decir, la equidad externa, se producen procesos de compensación interna en el sistema educativo, es decir, de equidad interna (Bolívar, 2012). A la larga, esto genera procesos de cooperación educativa y social que no solo son beneficiosos para los individuos que reciben dicha compensación, sino también para el conjunto de la sociedad, incluidos los más aventajados. Según Noguera (2002: 218), la teoría rawlsiana nos aporta ciertas ventajas respecto al utilitarismo y al igualitarismo doctrinario. En este sentido, fundamenta teóricamente cuáles son las desigualdades que una sociedad puede admitir y las razones que la justifican, y, al mismo tiempo, nos brinda una definición precisa del tipo de igualdad que deben sostener los denominados «igualitaristas». Además, aporta unos principios claros que pueden servir a nivel práctico para orientar la política social (Noguera, 2002). Por otro lado, la inclusión del principio de diferencia en la teoría rawlsiana hace que esta supere

1. La importancia de la teoría de Rawls reside en que, con la publicación de su libro Una teoría de la justicia, cambia el panorama ético y desafía al sistema dominante, el utilitarismo, reactualizando el concepto de justicia en base a la elección racional sustentada en principios de libertad y de oportunidad para todos (Ribotta, 2009). 
otras teorías de la justicia distributiva, ya que se establece que las desigualdades no deben permitirse, a no ser que se considere que estas podrían beneficiar a los individuos o a los grupos sociales menos aventajados (Gargarella, 1999; citado por Bolívar, 2005).

\section{Limitaciones y críticas de la justicia como compensación}

En el siguiente apartado, se examinarán las principales críticas que se han hecho a la justicia distributiva dentro del campo de la educación, se mostrarán algunos enfoques alternativos, se desarrollarán algunas de las principales limitaciones del concepto de igualdad de oportunidades y se expondrán las críticas que se han hecho a las políticas que partían de esta idea.

La principal crítica que surge a la teoría de Rawls es la capabilities approach, de Amartya Sen. Sen (1995) tacha la teoría de Rawls de idealista y lleva el plano de su análisis hacia la capacidad de realización. De este modo, la equidad se juzgará en términos de oportunidad de los individuos de obtener o realizar algo, y no tanto en su obtención o realización efectiva. Es por ello que, según Sen (1995), para medir la justicia que hay en una sociedad, esta se ha de analizar a partir de las capacidades de los ciudadanos para convertir determinados bienes en libertades. Esto, traducido al campo pedagógico, hace referencia al hecho de que la valoración de los procesos educativos no se podrá centrar solo en los bienes y en los servicios que se distribuyen en el interior de los itinerarios escolares, sino que también habrá que contemplar las condiciones de posibilidad que se generan en estos (Tarabini, 2006).

En el campo educativo, la primera crítica que se formula a la justicia distributiva es que, a menudo, cuando se aplica a la educación, se pone mucho énfasis en el qué, en cambio, se olvida de cuestiones importantes vinculadas al cómo, omitiendo así aspectos tan relevantes como los contenidos y la forma de la propia educación (Tarabini, 2006). En este sentido, Conell (1997) sostiene que distribución y contenido son inseparables debido a su propia naturaleza. No obstante, las llamadas "políticas distributivas», que se han asociado a la igualdad de oportunidades en el acceso al mundo académico, han tendido a tomar en consideración aquellos elementos de carácter extraescolar para comprender las desigualdades, obviando aquellos elementos internos al propio sistema pedagógico que, a menudo, ejercen una gran influencia sobre las desigualdades sociales (Casassús, 2003; Tarabini, 2006). Si bien es cierto que el rendimiento escolar del alumnado está influido por factores de origen social, existen otros elementos de carácter interno (elementos push out) vinculados a la manera en que funciona el sistema educativo que pueden disminuir o bien acentuar las desigualdades (Marchesi, 2000; Tarabini, 2006). Como ejemplo de estos mecanismos, encontramos la agrupación por niveles o la homogeneización de grupos, así como el trato diferencial y la clasificación basada en expectativas del profesorado, elementos que muestran resultados desiguales en cuanto a la integración y al desarrollo del alumnado (Escudero y Martínez, 2013). 
Una de las medidas que sirve para ilustrar las carencias de la justicia distributiva en el mundo de la pedagogía hace referencia a los programas de educación compensatoria. Estos surgieron en las décadas de 1960 y 1970 como una medida para paliar aquellas desigualdades de origen (Bolívar, 2005, 2012) que hacían que los individuos se ubicaran en una posición desigual frente a la educación y que no se habían podido resolver mediante la extensión de la escuela de masas (Tarabini, 2006). Estas políticas se empezaron a desarrollar en Estados Unidos, en el marco de la lucha contra la pobreza, y, de manera progresiva, se fueron extendiendo por todo el mundo como consecuencia de las reformas educativas (de la escuela comprehensiva), que pretendían garantizar «la transición a una escuela media o secundaria para todos (Francia, Bélgica, Suecia, Inglaterra)» (Bolívar, 2012: 34).

El objetivo de estas políticas compensatorias era mejorar las oportunidades educativas y contribuir a la igualdad de los recorridos del alumnado, lo que no era posible garantizar solo con el acceso a la escolarización de los alumnos, ya que había que compensar las desventajas de tipo social o cultural de los más desfavorecidos (Bolívar, 2012). A pesar de todo, según Conell (1997), estos planteamientos parten de una concepción del problema que es errónea en sí misma, pues entienden "que las desigualdades educativas son un problema solo de las minorías desfavorecidas, suponen que los pobres son culturalmente diferentes a la mayoría y contemplan la reforma educativa como un problema eminentemente técnico», con lo cual sitúan «la raíz del problema en los propios colectivos desfavorecidos o en las escuelas concretas que los atienden, produciendo una perversión de la situación» (Tarabini, 2006: 250). Para comprender la desigualdad educativa, Conell (1997) considera importante focalizar el análisis en la escuela, observar qué sucede dentro de la institución y cómo se desarrollan los procesos académicos, fijándose en aspectos tales como las relaciones que se producen en el seno del aula, el currículo, etc.

Ligado con esto, el mismo Bernstein (1999) hace una crítica a la educación compensatoria, porque pone el foco de su mirada en las carencias de los sectores sociales más desfavorecidos y omite completamente las formas de organización de la escuela y el contexto educativo de la misma. Por tanto, el problema de estas políticas es que parten del supuesto de que lo que explica el fracaso o el abandono del sistema educativo son las carencias familiares, que impiden que los individuos puedan aprovechar adecuadamente su paso por el centro docente y que, a la vez, crean nuevos conceptos pedagógicos, como, por ejemplo, la deficiencia cultural, para explicar estas pautas de comportamiento de los alumnos de determinados orígenes sociales frente a la escuela (Tarabini, 2006). Así pues, Bernstein (1999) nos aporta una nueva mirada que permite fijarnos en las desigualdades que se producen en los procesos académicos, profundizando en cuestiones como, por ejemplo, de qué modo se organiza, se transmite y se evalúa el conjunto de saberes que emanan de la institución pedagógica, mediante el análisis de los códigos sociolingüísticos y sus funciones. Posteriormente, otros autores se han fijado en aspectos que generan desigualdades en el mundo educativo, como la cultura del centro (Smyth y Hattam, 2002). 
Finalmente, enfatizando en algunos de los aspectos generadores de desigualdad, en una de sus últimas obras, Brighouse y Swift (2014) ponen sobre la mesa el papel de la familia como agente generador de igualdad o desigualdad en el proceso educativo, desigualdad que se da tanto en términos de elección de centro como en la titularidad del mismo o el soporte a la escolarización, entre otros factores. De este modo, los autores se plantean la siguiente cuestión: ¿ Hasta dónde debe llegar la capacidad de elección, es decir, la libertad individual de elegir sobre el futuro de sus hijos, cuando esta elección se contraponga al principio de igualdad y de equidad? $\mathrm{O}$, lo que es lo mismo: ¿Qué sucede cuando la promoción de los valores familiares requiere «sacrificar» principios necesarios para la consecución de la igualdad? Si bien los autores señalan algunas posibles respuestas, este campo aún presenta mucho margen de discusión.

En otra línea similar, según Satz (2007), la intervención de la política pedagógica debe asegurar oportunidades justas para la consecución de posiciones educativas y laborales por encima del mínimo existente. De este modo, incluye conceptos como el de equidad, asegurando que ningún grupo social debe ser relegado a una posición de segunda clase, con acceso solo a los centros docentes y a los puestos de trabajo inferiores, si bien, para dicha consecución, se asume de nuevo que las oportunidades justas no tienen por qué ser iguales, así como que existe cierto grado de desigualdad aceptable, aunque esta tenga límites.

Por último, hay que destacar la importancia de los principios que la justicia distributiva ha aportado y que, sin duda, han contribuido a facilitar la comprensión de un ámbito concreto de la desigualdad educativa (Tarabini, 2006). Sin embargo, en los próximos apartados, se intentará complementar esta visión con diversas aportaciones que van más allá de la perspectiva redistributiva, como son las de Conell (desde la teoría de la justicia curricular), Fraser (la justicia como reconocimiento) o Bell y Harnath (la justicia como participación).

\section{Justicia postdistributiva y equidad educativa}

En los apartados desarrollados hasta el momento, hemos podido analizar las diversas propuestas sobre la distribución o, más concretamente, sobre las diversas propuestas de política redistributiva aplicables y aplicadas a la educación, pasando por las concepciones más tradicionales meritocráticas y las aportaciones revisionistas del igualitarismo de Rawls, si bien la mayoría de autores contemporáneos, incluido el propio Rawls, ha llegado al consenso de que la (re)distribución, per se, es insuficiente.

Esta idea se plasma principalmente en los postulados del igualitarismo predistributivo, que, según autores como Heckman (2006) o Hacker y Pierson (2010), se basaría en el precepto de que, para reducir la desigualdad, no podemos limitarnos a intervenir (ex post) sobre sus efectos, sino que debemos actuar (ex ante) sobre sus causas. Este enfoque predistributivo de la justicia social, de marcado carácter estructuralista, estaría enfocado a la prevención 
de la reproducción de las desigualdades en base a políticas que identifiquen y corrijan las deficiencias que las causan (Hacker y Pierson, 2010).

Sin embargo, nuestra propuesta pretende sistematizar otras dimensiones que, si bien están directamente relacionadas con la distribución y, por ende, con la predistribución, se centran en aquellos elementos subjetivos que quedan habitualmente más allá de esta. Algunos ejemplos de este tipo de dimensiones serían los siguientes: la individualización de la justicia educativa (no tanto entendida como la compensación de las desigualdades, sino como la adecuación del trato y los contenidos), la representación de los diferentes agentes pedagógicos o el reconocimiento de los mismos.

Así pues, podríamos categorizar este enfoque postdistributivo como un cajón de sastre que englobaría aquello no "atendible» desde la justicia predistributiva o que lo repensaría desde una lógica diferente. Se podría definir como un enfoque que, sin ir a las causas estructurales de la desigualdad, pretendería revertir ciertos aspectos de la misma a partir de intervenciones micro en el seno de las instituciones. Además, esta perspectiva diferiría de la predistributiva en el hecho de que incorporaría la subjetividad de los agentes y repensaría viejas dimensiones de las esferas cultural e ideológico-política. Este enfoque, pues, haría referencia a aquellos elementos de la micropolítica que deberían permitir ampliar los conceptos de equidad, justicia e igualdad dentro de las instituciones educativas más allá de la compensación (redistribución), y sin la necesidad de repensar estructuralmente la propia institución (predistribución).

En esta línea, dicha propuesta pretende incidir, sin olvidar la lógica propia de la predistribución, en aquellos elementos intrínsecos del sistema pedagógico o de la institución escolar que envuelven a las desigualdades sociales y educativas. Sin embargo, del mismo modo que sucede en otros campos como el de los «bienes comunes», creemos que, en el campo de la educación, se debe profundizar en este debate y pensar nuevos enfoques y nuevas teorías de la justicia distributiva que combinen elementos de la predistribución y de la postdistribución (Laín, 2014).

Más concretamente, en este último apartado, nos centraremos en aquellas visiones que han apostado por la consecución de la equidad en dos de los ámbitos educativos, al margen de los recursos, más relevantes para la política pedagógica: la atención a la diversidad y el currículo. Para finalizar, atenderemos a la importancia del reconocimiento y la representación en el campo educativo como dos de los elementos imprescindibles para la consecución de la equidad.

\subsection{Justicia pedagógica. La igualdad y la justicia en la atención y el trato}

Un primer elemento interesante a considerar dentro de este enfoque es la política de atención a la diversidad y su incidencia en la consecución de la equidad educativa. De ahora en adelante, a esta dimensión, la llamaremos «justicia pedagógica». Históricamente, el énfasis en esta se orientó, en un primer momento, a la lucha contra el fracaso escolar. Posteriormente, se desplazó a la lucha contra la exclusión educativa y social (Escudero y Martínez, 2013). 
La idea de fondo se basa en la ruptura de los ya mencionados principios de igualdad estricta, considerando que la igualdad formal como tal es injusta, con el objetivo de generar situaciones de refuerzo ahí donde se detecta desventaja educativa (Bolívar, 2012). Como práctica social, se caracteriza por el poder y por el deseo de enseñar y aprender en la diferencia, idea ya avanzada en la teoría de la justicia social de Rawls, que se constituye como fundamento de calidad y que se despliega desde la equidad, sin olvidar el mérito (Lamas, 2006). El referente conceptual de esta teoría de la justicia es el de igualdad en la diversidad. Este precepto resulta importante, ya que cambia la concepción del «todo es igual» e introduce la diversidad y las diferencias como elementos a tener en consideración en el marco de la justicia aplicada en los centros educativos ${ }^{2}$.

Dentro de esta dimensión, podríamos destacar tres ámbitos: las políticas de compensación (que se han tratado en el apartado anterior y que se corresponderían a una visión predistributiva de la equidad y la justicia social), la lucha contra la exclusión educativa y la maximización de las oportunidades de éxito educativo.

En primer lugar, la lucha contra la exclusión trata de constituirse como un mecanismo de atención para los grupos en riesgo, tratando de «mejorar la suerte de los vencidos en la competencia educativa y social». Este concepto apuesta por una equidad que vaya más allá de la igualdad y que garantice a la ciudadanía un nivel básico de bienes sociales y de sentimiento de inclusión (Bolívar, 2012).

En segundo lugar, la maximización de oportunidades de éxito busca la adaptación de la heterogeneidad social con el objetivo de potenciar a cada uno de esos individuos heterogéneos. Es desde dicha línea que se desarrolla el concepto de escuela inclusiva y la lógica de maximización de oportunidades de éxito. El crecimiento de dispositivos de atención a la diversidad, ya sea para alumnado con necesidades educativas especiales (de carácter físico o psicológico) o especificas (de carácter socioeconómico), para atender a las minorías étnicas y sociales o para potenciar a los alumnos de altas capacidades, evoca paradójicamente a la equidad como objetivo.

Ambos ámbitos parten de la base de la atención a la diversidad como clave para articular la justicia social en la diferencia. Según Essomba (2003), entenderemos esta atención a la diversidad como un conjunto de estrategias educativas, y también pedagógicas, que conducen a satisfacer, en un marco integral, las necesidades educativas de todas las personas sin renunciar a un proyecto didáctico común. Es por ello que, cuando hablamos de atención a la diversidad, estamos hablando inevitablemente de una intervención y no de una «realidad neutral». Si bien se debe vigilar con los efectos perversos producibles bajo objetivos bienintencionados que puedan erigir un sistema dual y desigual en sus efectos.

2. Hay que tener en cuenta las aportaciones de Walzer (1993) en la diferenciación entre igualdad simple y compleja y su aplicación al campo de la justicia educativa. La atención a la diversidad trata de romper con la igualdad simple, ya que esta multiplica las desigualdades a pequeña escala. 
Un elemento importante a considerar derivado de este proceso es el efecto Pigmalión o el de profecía autocumplida, a través del cual estos individuos interiorizan la diferencia que les caracteriza influyendo en su conducta y en sus resultados posteriores (Rosenthal y Jacobsen, 1968).

Es por ello que, en un sistema de atención a la diversidad sin una percepción clara de la función y los objetivos de sus dispositivos, se pueden dar procesos de reducción de las exigencias y las expectativas por parte de los profesionales que les atienden, además de generar el mismo proceso en los jóvenes, lo cual afectaría a la equidad y a la justicia que precisamente con estos mecanismos se pretendía obtener (Lamas, 2006)

En esta concepción de la justicia educativa y pedagógica, la justicia va ligada a los principios de libertad-igualdad y equidad-diferencia, enfatizando en la concepción equitativa e introduciendo elementos sustanciales de diferencia, pero sin olvidar ciertas vinculaciones que permitan formular criterios para determinar qué situaciones son estimadas justas e injustas.

$\mathrm{Si}$, en la traducción de este ideario, focalizamos en las medidas concretas de atención a la diversidad que proclama la LOGSE — primer intento de normativizar dichos preceptos en el Estado español- el resultado es el surgimiento del concepto de necesidad educativa especial y el de atención a la diversidad. Ello posibilitó, aunque fuese de manera parcial, el surgimiento de medidas de carácter predistributivo y redistributivo, como el establecimiento de programas de integración, compensación y adaptación educativa, estableciendo medidas legislativas concretas y proporcionando recursos más adecuados para enfrentar de forma más equitativa estas «nuevas» situaciones didácticas.

En definitiva, el objetivo último de la justicia pedagógica es el de superar las imágenes discriminatorias, la asociación de la diversidad con la pobreza, el desmérito y la inutilidad, para colaborar en la formulación de preceptos macropolíticos que posibiliten una escuela y una educación más justas e igualitarias en el marco de la diversidad.

\subsection{Justicia curricular. La igualdad y la justicia en la construcción del curriculo}

El segundo elemento que analizaremos dentro de este conjunto de propuestas es aquel referente a la justicia curricular. El currículo es uno de los elementos más importantes en el análisis de los sistemas educativos (Apple, 1979). La pregunta que surge al respecto es la siguiente: ¿qué implicaciones, en términos de justicia social, genera un currículo igual para todos los alumnos? Según Bernstein (1999), el currículo es fruto de un proceso de selección, clasificación y distribución del conocimiento en base a la distribución del poder en una determinada sociedad. Esto demuestra, en parte, cómo el sistema en sí y su propia organización generan una serie de desigualdades intrínsecas. La fuerza de ese currículo reside en su capacidad de presentarse como conocimiento objetivo y neutral, hegemónico, escondiendo la relación bidireccional que se produce entre conocimiento y sociedad (la estructura social determina el conocimiento que se produce, mientras que la producción de conocimiento tiene consecuen- 
cias e impactos sociales) y despolitizando la acción de este sobre la justicia social bajo estos supuestos de neutralidad y objetividad (Apple, 1979). Ligando estos elementos con la idea de justicia de Rawls, Conell establece tres ámbitos de justicia curricular en la construcción del currículo contrahegemónico.

El primero es el de favorecer los intereses de los menos favorecidos. Este planteamiento, dice Conell, debe realizarse en base a posicionamientos de carácter socioeconómico, empoderando a los más desfavorecidos económica y culturalmente, como si de una política redistributiva se tratara, pero también en base a cuestiones de género y etnia, generando sinergias con las políticas de reconocimiento (Conell, 2009), tal y como veremos en el apartado posterior. Por último, para que la construcción de este currículo "contrahegemónico" encarne dichos intereses, este debe ser diseñado por y para materializar los intereses de los menos favorecidos, así como para enaltecer sus perspectivas y aportaciones. Esto requiere, como veremos a continuación, la participación activa de profesionales cualificados, preferiblemente de ámbitos multidisciplinares (atención a la diversidad, educación compensatoria, etc.) y, directa o indirectamente, de los destinatarios (principio de representación). En términos de Michael Apple, si bien este proceso contrahegemónico es arduo, generaría espacios críticos que presumiblemente impactarían en el currículo oculto y su visión tácitamente hegemónica (Apple, 1979).

El segundo punto clave en la justicia curricular es la participación y la escolarización común. Esto requiere que el currículo contrahegemónico no se acabe convirtiendo en independiente ni unidimensional. Solo si se establece un currículo contrahegemónico en el seno de la institución educativa, que, a su vez, tenga en cuenta las múltiples caras de la desigualdad, se podrá desinstitucionalizar y desinstrumentalizar la misma (Conell, 2009). Esta desinstrumentalización de la práctica educativa desigual debería introducir, a su vez, elementos de desarrollo del conocimiento, de las habilidades sociales, de las actitudes cívicas y de los valores democráticos, capacitando a los alumnos a intervenir en la vida social como ciudadanos activos, empezando con la participación en el propio currículo y en las decisiones colectivas del aula.

El tercero ámbito es el de la producción histórica de la igualdad, que implica a todo el conjunto de relaciones sociales que operan dentro del sistema educativo (Conell, 1997). Con este principio, se rompe con la concepción de la igualdad como un elemento estático, poniendo énfasis en la necesidad de realizar un análisis constante de la producción de igualdad.

Si aplicamos estos términos a la actualidad, veremos que, desde las instituciones pedagógicas con mayor reconocimiento internacional —entre ellas, la Unión Europea—, se han redefinido los objetivos educativos en términos de competencias. De este modo, se entiende que, al finalizar el periodo educativo obligatorio, los alumnos deben haber incorporado una serie de competencias necesarias para su vida en comunidad. En palabras de Conell (2009), aquí está la base de un currículo común que se debe ofrecer a todos los estudiantes como una cuestión de justicia social y que tiene que descartar cualquier tipo de selección, de evaluación competitiva, de mecanismos de agrupamiento y de 
clasificación en la escolarización (fuera del currículo común, es otro debate), ya que estos mecanismos diferencian las ofertas $y$, por tanto, favorecen a unos ciudadanos sobre otros.

$\mathrm{Si}$, a esta lógica, añadimos la idea anterior de que las desigualdades serán aceptables siempre y cuando contribuyan a mejorar a aquel alumnado considerado más desaventajado, obtenemos que, en este paradigma, un sistema educativo es justo no necesariamente cuando reduce las desigualdades internas, sino cuando garantiza, para los estratos más bajos, la adquisición de aquellas competencias entendidas como básicas (Dubet y Duru-Bellat, 2007). De este modo, la escuela tiene que hacer frente a una contradicción entre distintos principios alrededor de la justicia: «afirma a su vez la igualdad de inicio de todos los alumnos y su capacidad para producir desigualdades justas a partir de sus clasificaciones y jerarquías» (Dubet, 2010: 17). Así pues, la cuestión alrededor de la cual debería reflexionar la institución pedagógica es la siguiente: ¿cómo producir desigualdades que sean justas?

Sin embargo, no se pueden obviar los aspectos negativos vinculados al back to basics (priorización del contenido y las habilidades consideradas básicas o fundamentales) de una concepción meramente competencial de la educación. Es por ello que esta visión ha sido ampliamente criticada por su base epistemológica subyacente a una visión mercantilista de la educación o por su falta de visión crítica, entre otros aspectos (Del Rey y Sánchez-Parga, 2011).

Finalmente, no se trataría tanto de reducir la exigencia y los contenidos bajo la misma lógica de evitar la reificación o la generación de un subsistema educativo para los desfavorecidos, sino de eliminar posibles condicionantes negativos que puedan generar situaciones de riesgo o de vulnerabilidad social (Bolívar, 2012). En esta línea, se busca la ruptura de la lógica credencialista del currículo ordinario, para así evitar el efecto desalentador que este produce sobre las expectativas del profesorado, pero también sobre las del propio alumnado desfavorecido. Uno de los ejemplos más evidentes lo observamos en el uso que se da, en estos itinerarios curricularmente diferenciados, de mecanismos como la repetición. Finalmente, como dice Conell 2009: 6): «las prácticas curriculares son injustas cuando reducen la capacidad de las personas para mejorar su mundo». En esta línea, retomando la tesis clásica de Apple, el resultado de la no interpretación critica del currículo será la reproducción cultural y económica de las relaciones de clase de las sociedades desiguales (Apple, 1979).

\subsection{Más allá de la distribución (y la redistribución). El reconocimiento y la representación}

Finalmente, como consecuencia, por un lado, de la crisis del estado de bienestar keynesiano (EBK) y sus políticas sociales de redistribución económica (tradicionalmente impulsadas por los partidos de cariz socialdemócrata), y, por otro y todavía más importante, del surgimiento, a partir de la segunda mitad del siglo xx, de nuevos movimientos sociales, como, por ejemplo, el 
movimiento feminista, se colocaron las políticas de reconocimiento encima de la mesa (Fraser, 2008; Bolívar, 2012). Esto ponía de manifiesto la idea de que la justicia social, en términos generales, pero también en su aplicación en el ámbito educativo, no puede ser explicada a través de una única dimensión, sino que, al contrario, está compuesta por una serie de esferas.

Estas esferas, además de la económica, sobre la cual se ha basado la mayoría de estudios previos exigiendo medidas predistributivas y redistributivas al respecto, son la cultural, que requiere de medidas de reconocimiento, y la política, que se concreta a través de la representación y la participación (Fraser y Honneth, 2005).

En este sentido, la esfera relacionada con la igualdad de reconocimiento incluiría la dignidad en el trato, el reconocimiento cultural, así como la no discriminación por motivos de género, etnia o raza. En esta perspectiva, la justicia educativa y social vendrá delimitada por las prácticas que posibiliten el reconocimiento mutuo, la atención afectiva, la igualdad jurídica y la estima social. Autores como Fraser y Honneth (2005) detectan, pues, situaciones colectivas de riesgo en las que no está en juego la distribución de bienes, sino una ausencia de afectos y de estima social, que inciden con la misma negatividad. En este sentido, es importante señalar la contribución realizada por Reay (2000) con el desarrollo del concepto de capital emocional y su relación con el éxito o el fracaso educativo. Por otro lado, cuando se incorpora el reconocimiento cultural en la concepción de la justicia educativa y social, la igualdad de oportunidades es desplazada por el reconocimiento de las identidades culturales, lo cual genera nuevas dimensiones de debate y nuevas propuestas en forma de políticas (Fraser y Honneth, 2005). La política de la diferencia (Young, 2000), ya sea en su versión de género o en su versión multicultural, es un ejemplo de esta otra cara de las políticas de igualdad educativa que reclaman la afirmación positiva de las diferencias.

Sin embargo, sería un error tratar el reconocimiento como una esfera independiente de la predistribución. Mientras una perspectiva hace énfasis en las condiciones materiales, la otra hace referencia a elementos culturales, en ningún caso excluyentes unos de otros. Históricamente, se ha tendido a supeditar las segundas a las primeras, considerando que las condiciones de igualdad son más determinantes que las condiciones culturales. Sin embargo, se pueden atribuir determinados aspectos básicos de la desigualdad educativa a estas desigualdades culturales, por ejemplo, como veíamos antes, a través de currículos dominantes culturalmente 3 (Fraser y Honneth, 2005). El peligro, para autores como Power (2008), reside entonces en la naturalización de este fracaso, siendo más necesario que nunca el uso de este tipo de políticas de reconocimiento, tanto en términos culturales como de clase.

3. No se pretende negar la importancia de las condiciones materiales de igualdad, sino poner atención a las condiciones culturales. Si bien las segundas no podrán incidir en la desigualdad social con el mismo impacto que las primeras, pueden ayudar a reducir ciertas desigualdades internas (en el sistema educativo) y mejorar la experiencia académica de los menos favorecidos. 
Pero, para que esta dicotomía de concepciones — la redistributiva y la de reconocimiento- funcionen de forma integrada, se requiere una tercera dimensión de la igualdad: la representación (Fraser, 2008, en Bolívar, 2012). Esta dimensión hace referencia a la dimensión política y a la igualdad de participación. Según Fraser, no hay redistribución ni reconocimiento sin representación. Es decir, se requieren consensos sociales para que se dé una participación igualitaria por parte de todos en la vida social y educativa. Es, pues, desde la tríada de las tres erres (redistribución, reconocimiento y representación) que se puede generar una lucha por la igualdad educativa que lleve a acciones afirmativas y transformadoras en clave de justicia social (Bolívar, 2012).

Finalmente, las teorías de la justicia como representación y como participación son interesantes de incorporar en las políticas educativas que quieran hacer frente a las desigualdades y reducir el fracaso y el abandono escolares, puesto que pueden contribuir a aumentar la adhesión académica de los alumnos más desfavorecidos (Bonal y Tarabini, 2010).

\section{Conclusiones}

En el actual contexto de crisis, vemos como emerge nuevamente el debate sobre la justicia y las desigualdades, que se acentúa como consecuencia de las crecientes tasas de fracaso y abandono escolares que afectan a los países del sur de Europa, como España (24,9\%), Malta (22,6\%), Portugal (20,8\%) o Italia $(17, \%)^{4}$. Ante esta problemática social, han resurgido viejas preguntas, por ejemplo: ¿cómo reducir la desigualdad social y educativa? ¿Qué desigualdades son justas? ¿En qué tiene que haber igualdad? ¿Y entre quién? Huelga decir que las respuestas a estas y a otras cuestiones tienen implicaciones profundas en la manera de concebir y diseñar las políticas educativas.

A lo largo del presente ensayo, se han abordado diferentes teorías de la justicia que se han intentado aplicar en el campo de la educación, con lo cual hemos visto una panorámica del estado de la cuestión sobre la teoría social normativa en dicho ámbito. En este sentido, hemos podido comprobar que las maneras de concebir la igualdad de Rawls, Conell, Fraser o Harnath, así como las implicaciones que tiene en términos de políticas educativas, son muy diferentes. No obstante, hay que reconocer que esta es una primera aproximación a la temática tratada, en la cual no se han podido englobar todos los debates que existen en la actualidad. Reconociendo, pues, las limitaciones del presente estudio, se ha querido situar en él algunas de las teorías de la justicia más relevantes, mostrando sus restricciones, así como también las posibles aplicaciones en el ámbito de la educación. De cualquier modo, si algo podemos subrayar es que, como dice Tarabini (2006), la educación debe entenderse como un ámbito

4. Early school leavers from education and training (CE, 2012: 5). Fuente: Comisión EuroPEA (2012), Europe 2020 target: Early leavers from education and training [en línea], <http:// ec.europa.eu/europe2020/pdf/themes/29_early_school_leaving.pdf> [Consulta: 7 enero 2015]. 
que posibilita la aplicación de la justicia social. No se trata del único, pero sí resulta muy relevante. Además, la importancia de ello no debe entenderse como exclusiva de aquellos que usan y disfrutan del bien (la educación), en este caso los estudiantes y sus familias, sino como un espacio que facilita el bienestar colectivo, ya que sus efectos repercutirán en el conjunto de la sociedad.

En segundo lugar, si bien se trata de una idea ya ampliamente extendida entre los sociólogos de la educación, es interesante remarcar cómo conceptos moralmente deseables como la igualdad, al aplicarlos a dimensiones concretas como es el caso del campo educativo, pasan a ser conceptos necesarios pero insuficientes, cuyo valor absoluto deja de ser en cierto modo anhelable, por las repercusiones no deseadas que el mismo genera. En ciertos casos, tal como hemos remarcado durante todo el artículo, la igualdad stricto sensu se convierte en el mayor escollo para la atención y el desarrollo de la diversidad. Los currículos adaptados y la atención a la diversidad, más allá de las meras políticas compensatorias, constituyen un primer paso de introducción activa de elementos de justicia social en educación, más allá de la igualdad de oportunidades de acceso, si bien aún queda perfeccionar estos mecanismos. Es necesario evitar su extrema rigidez y la generación de un subsistema educativo de segunda clase, el cual, según autores como Satz (2007), supondría la pérdida de las oportunidades justas, necesarias para la consecución de la equidad educativa y laboral.

Como hemos podido ver, hablar hoy de justicia y de igualdad es una tarea ardua, pues hacer una aproximación rigurosa implica necesariamente superar las nociones de sentido común de estos conceptos y analizar los principios y los significados que se derivan de las diferentes perspectivas. En el presente estudio, hemos podido ver diversas aproximaciones a las principales teorías acerca de la justicia distributiva. Después de analizar el sentido de esta teoría en el ámbito académico y después de ver sus limitaciones, resulta evidente la necesidad de entender la justicia de manera integral, es decir, como sinónimo de equidad educativa, mediante una noción que nos permita hacer frente a las desigualdades de acceso, de proceso (y, por lo tanto, mirando en el interior de la escuela, atendiendo a cuestiones de reconocimiento y participación), así como de los resultados.

En la siguiente tabla, se intenta sintetizar las diferentes teorías de la justicia en base a tres elementos: su impacto en términos de igualdad y en términos de equidad, así como los posibles outputs que se derivan de la implementación de dichos enfoques en forma de políticas o medidas educativas concretas.

Finalmente, a modo de prospectiva, querríamos remarcar ciertas líneas para futuras investigaciones al respecto. En primer lugar, una acción interesante sería analizar el efecto de los cambios legislativos de las últimas reformas educativas en relación con la igualdad, la equidad y la justicia social. ¿Caminamos hacia un sistema más justo y equitativo o, por el contrario, cada vez nos alejamos más de él? ¿Qué medidas concretas se están generando al respecto? ¿Por qué estas y no otras? Esta reflexión inicial nos tendría que proporcionar herramientas para pensar de manera crítica alrededor de la política educativa 
Tabla 1. Síntesis de las teorías de la justicia y sus impactos

\begin{tabular}{|c|c|c|c|}
\hline & Igualdad & Equidad & Resultado \\
\hline $\begin{array}{l}\text { Justicia } \\
\text { meritocrática }\end{array}$ & $\begin{array}{l}\text { Igualdad de } \\
\text { oportunidades. }\end{array}$ & $\begin{array}{l}\text { Programas compensa- } \\
\text { torios residuales. }\end{array}$ & $\begin{array}{l}\text { Expansión de los siste- } \\
\text { mas educativos (acceso } \\
\text { y oportunidades univer- } \\
\text { salizados). }\end{array}$ \\
\hline $\begin{array}{l}\text { Justicia distributiva } \\
\text { (rawlsiana) }\end{array}$ & $\begin{array}{l}\text { Igualdad } \\
\text { compensatoria. }\end{array}$ & Principio de diferencia. & $\begin{array}{l}\text { Expansión de los pro- } \\
\text { gramas compensatorios } \\
\text { (ruptura del mérito). }\end{array}$ \\
\hline $\begin{array}{l}\text { Justicia } \\
\text { pedagógica }\end{array}$ & $\begin{array}{l}\text { Igualdad en la } \\
\text { diversidad. }\end{array}$ & $\begin{array}{l}\text { Política de } \\
\text { compensación. }\end{array}$ & $\begin{array}{l}\text { Implementación de polí- } \\
\text { ticas de atención a la } \\
\text { diversidad. }\end{array}$ \\
\hline Justicia curricular & $\begin{array}{l}\text { Igualdad en el currículo } \\
\text { y en el aprendizaje. }\end{array}$ & $\begin{array}{l}\text { Política de empodera- } \\
\text { miento (materializar } \\
\text { intereses de los } \\
\text { desfavorecidos). }\end{array}$ & $\begin{array}{l}\text { Construcción del currícu- } \\
\text { lo «contrahegemónico». }\end{array}$ \\
\hline $\begin{array}{l}\text { Justicia en el } \\
\text { reconocimiento }\end{array}$ & $\begin{array}{l}\text { Igualdad en el trato y } \\
\text { en el reconocimiento } \\
\text { cultural. }\end{array}$ & $\begin{array}{l}\text { Política de la diferencia } \\
\text { (no discriminación } \\
\text { étnica, de género, etc.). }\end{array}$ & $\begin{array}{l}\text { Reconocimiento de } \\
\text { las dimensiones de la } \\
\text { multiculturalidad y la } \\
\text { igualdad de género en la } \\
\text { educación. }\end{array}$ \\
\hline $\begin{array}{l}\text { Justicia } \\
\text { representativa }\end{array}$ & $\begin{array}{l}\text { Igualdad en la } \\
\text { participación. }\end{array}$ & Política de participación. & $\begin{array}{l}\text { Surgimiento de meca- } \\
\text { nismos de participación } \\
\text { democrática en la } \\
\text { escuela. }\end{array}$ \\
\hline
\end{tabular}

Fuente: elaboración propia.

y las nociones de justicia y de igualdad, al mismo tiempo que nos tendría que permitir razonar qué criterios y/o principios tendrían que regir determinadas políticas educativas que pretenden combatir la desigualdad y, muy especialmente, aquellas destinadas a hacer frente al fracaso y al abandono escolares.

\section{Referencias bibliográficas}

Apple, M.W. (1979). Ideology and Curriculum. Londres: Routledge.

Bernstein, B. (1999). "Una crítica a la educación compensatoria». En: Fernández Enguita, M. (ed.). Sociología de la educación. Barcelona: Ariel.

Boвbio, N. (1993). Igualdad y libertad. Barcelona: Paidós.

Bolívar, A. (2005). "Equidad educativa y teorías de la justicia». Revista Electrónica Iberoamericana de Calidad, Eficacia y Cambio en Educación (REICE) [en línea], 3 (2), 42-69. <http://www.rinace.net/arts/vol3num2/art4.htm>.

- (2008). "Competencias básicas y ciudadanía». Caleidoscopio: Revista del CEP de Jaén [en línea], 1, 4-32. <http://revista.cepjaen.es/numero_01/articulos.html>.

- (2012). "Justicia social y equidad: Una revisión actual». Revista Internacional de Educación para la Justícia Social (RIEJS), 1 (1), 9-45.

Bonal, X. (2005). Sociología de la educación: Una aproximación critica a las corrientes contemporáneas. Barcelona: Paidós. 
Bonal, X. y Tarabini, A. (2010). Ser pobre en la escuela: Habitus de pobreza y condiciones de educabilidad. Buenos Aires: Miño y Dávila.

Brighouse, H. y Swift, A. (2014). Family Values: The Ethics of Parent-Child Relationships. Princeton, NJ: Princeton University Press.

Casassús, J. (2003). La escuela y la desigualdad. Santiago de Chile: LOM Ediciones.

García Cívico, J. (2006). La tensión entre mérito e igualdad: El mérito como factor de exclusión. València: Universitat de València. Tesis doctoral.

Conell, R.W. (1997). Escuelas y justicia social. Madrid: Morata.

- (2009). "La justicia curricular». Foro latinoamericano de políticas educativas, año 6, 27, julio. Buenos Aires: LPP (Laboratorio de Políticas Públicas).

Dubet, F. (2010). Decadència de la institució escolar i conflictes entre principis. Barcelona: Fundació Jaume Bofill.

- (2011). Repensar la justicia social: Contra el mito de la igualdad de oportunidades. Buenos Aires: Siglo XXI Editores.

Dubet, F. y Duru-Bellat, M. (2004). «Qu'est-ce qu'une école juste». Revue Française de Pédagogie, 146, 105-14.

- (2007). «What Makes for Fair Schooling?». En: Duru-Bellat, M.; Teese, R. y LAMв, S. (eds.). Education and Equity: International Perspectives on Theory and Policy, 3, 275-91. Dordrecht: Springer.

Escudero, J.M. y Martínez, B. (2013). «Las políticas de lucha contra el fracaso escolar: ¿Programas especiales o cambios profundos del sistema y la educación?». Revista de Educación, número extraordinario, 174-19.

Essomba, M.A. (2003). Els discursos sobre atenció a la diversitat en la comunitat educativa a Catalunya. Universitat Autònoma de Barcelona. Tesis doctoral.

Fernández Enguita, M. (1998). "La política educativa y las desigualdades de clase, genero y etnia». En: Alvarado Pérez, E. (coord.). Retos del Estado de Bienestar en España a finales de los noventa. Madrid: Tecnos.

- (2001). Educar en tiempos inciertos. Madrid: Morata.

- (2011). «Del desapego al desenganche y de éste al fracaso escolar». Propuesta Educativa, 1 (35), 85-94.

Fraser, N. (2008). Escalas de justicia. Barcelona: Herder.

Fraser, N. y Honneth, A. (2005). ¿Redistribución o reconocimiento?: Un debate politico-filosófico. Madrid: Morata.

Gallego Duque, L. (2014). Mecanismos causales de la educación y la pobreza: Aplicación a los casos de Medellín (Colombia) y Belo Horizonte (Brasil). Bellaterra: Universitat Autònoma de Barcelona. Tesis doctoral.

Gargarella, R. (1999). Las teorías de la justicia después de Rawls. Barcelona: Paidós.

Hacker, J.S. y Pierson, P. (2010). Winner-Take-All Politics: How Washington Made the Rich Richer-and Turned Its Back on the Middle Class. Nueva York: Simon \& Schuster.

Heckman, J. (2006). Investing in Disadvantaged Young Children is an Economically Efficient Policy. Comunicación presentada al Committee for Economic Development / The Pew Charitable Trusts / PNC Financial Services Group Forum on «Building the Economic Case for Investments in Preschool». Nueva York.

LAÍn, B. (2014). "Bienes Comunes y Justicia (pre)Distributiva». XI Jornades Internacionals de Filosofía Politica: Velles i Noves Formes d'Acció Política. Barcelona: Universitat de Barcelona.

Lamas, A.M. (2006). «Justicia pedagógica y atención a la diversidad». Rev. Complut. Educ., 17 (2), 51-56. Buenos Aires. 
Marchesi, A. (2000). "Un sistema de indicadores de desigualdad educativa». Revista Iberoamericana de Educación, 23.

Merton, R. (1948). "The Self-Fulfilling Prophecy». The Antioch Review [en línea], 8 (2), 193-210. <http://dx.doi.org/10.2307/4609267>.

Noguera, J.A. (2002). «Necrológica John Rawls». Papers: Revista de Sociologia, 68, 215-218.

Power, S. (2008). «How should we respond to the continuing failure of compensatory education?». Orbis Scholae [en línea], 2 (2), 19-37. <http://www.orbisscholae.cz/ archiv/2008/2008_2_02.pdf>.

Puyol, Á. (2007). «Filosofía del mérito». Contrastes: Revista Internacional de Filosofía, $12,169-287$.

Rawls, J. (1971). Teoría de la justicia. Madrid: Fondo de Cultura Económica, 1979.

REAY, D. (2000). «A useful extension of Bourdieu's conceptual framework?: Emotional capital as a way of understanding mothers' involvement in their children's education?». The Sociological Review [en línea], 48, 568-585. <http://dx.doi.org/10.1111/1467-954X.00233>.

Rey, A. del y Sánchez-PARga, J. (2011). "Crítica de la educación por competencias». Universitas: Revista de Ciencias Sociales y Humanas. UPS-Ecuador, 15, julio-diciembre, 233-24.

Riвотта, S. (2009). John Rawls, sobre (des)igualdad y justicia. Madrid: Dykinson.

Rosenthal, R. y Jacobsen, L. (1968). Pygmalion in the classroom: Teacher expectation and pupils' intellectual development. Nueva York: Holt, Rinehart and Winston.

Satz, D. (2007). "Equality, Adequacy, and Education for Citizenship». Ethics: An International Journal, of Social, Political, and Legal Philosophy [en línea], 117 (4), 623-648. $<$ http://dx.doi.org/10.1086/518805>.

Sen, A. (1995). Nuevo examen de la desigualdad. Madrid: Alianza Editorial.

Smyth, J. y Наттам, R. (2002). "Early School Leaving and the Cultural Geography of High Schools». British Educational Research Journal [en línea], 28, 375-397. <http://dx.doi.org/10.1080/01411920220137458>.

Tarabini, A. (2006). "Com analitzar la justícia dels sistemes educatius?: Una aproximació teòrica al concepte de justícia dins l'educació». Temps d'Educació, 31, 241-258.

Tedesco, J.C. (2004). «Igualdad de oportunidades y política educativa». Cadernos de Pesquisa [en línea], 34 (123), 555-572. <http://dx.doi.org/10.1590/s0100-15742004000300003>.

Walker, M. y Unterhalter, E. (eds.) (2007). Amartya Sen's Capability Approach and Social Justice in Education. Londres: Palgrave.

Walzer, M. (1993). Las esferas de la justicia: Una defensa del pluralismo y la igualdad. México: Fondo de Cultura Económica.

Young, I.M. (2000). Justicia y politica de la diferencia. Madrid: Cátedra.

Young, M.D. (1961). The rise of the meritocracy. 1870-1933: An essay on education and equality. Londres: Penguin Books. 